Editorial

\title{
Quality Improvement Initiatives in Health Sector in India: Need of the Hour
}

\author{
Dr. Charu
}

Assistant Professor, Department of Community Medicine, ESI Medical College \& Hospital, Faridabad, Haryana.

DOI: https://doi.org/10.24321/2454.325X.201920

I $\quad \begin{array}{lllll}\mathbf{N} & \mathbf{F} & \mathbf{O}\end{array}$

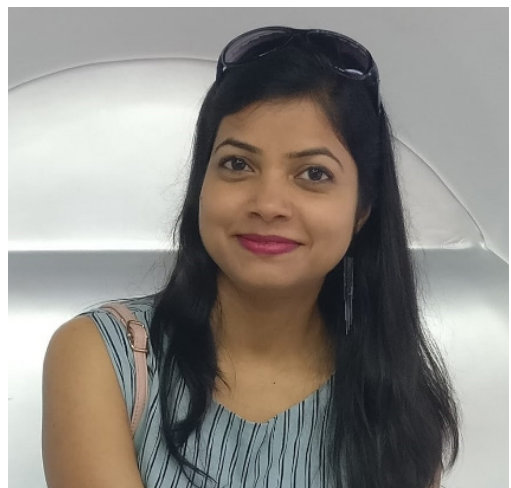

E-mail Id: drcharu2017@gmail.com

Orcid Id:

https://orcid.org/0000-0001-9069-5173

How to cite this article:

Charu. Quality Improvement Initiatives in Health Sector in India: Need of the Hour. Int J Preven Curat Comm Med 2019; 5(3): 1-2.
$\begin{array}{llllllll}\mathbf{A} & \mathbf{B} & \mathbf{S} & \mathbf{T} & \mathbf{R} & \mathbf{A} & \mathbf{C} & \mathbf{T}\end{array}$

Poor quality of health care services is one of the key barriers towards achievement of Sustainable Development Goals (SDGs). Despite significant improvement in coverage of health care services, the proportionate reduction in maternal and newborn mortality has not been observed which is due to inadequacies in quality of health care services. As per latest figure, only 11 states have achieved the National Health Policy Goal of Maternal Mortality Rate (MMR) less than 100 per lakh live births. States like Uttar Pradesh and Madhya Pradesh has seen rise in maternal mortality which is worrisome and making the situation worse. The key challenges are inadequate quality of health care, absence of an assured service delivery provision and poor involvement of community.

To improve the quality in health sector, Government of India recently launched Surakshit Matritva Aashwasan (SUMAN) initiative in October 2019 which aims for assuring respectful and dignified health care at no cost to the beneficiaries. Additionally, there will be zero tolerance for denial of health services to any woman or newborn irrespective of caste, religion, socio -conomic status, geographical region etc. at any public health facility leading to end all preventable newborn and maternal deaths. This will provide an overall positive birthing experience to everyone. The objective of SUMAN are to provide high quality medical and emergency services and referrals, to create a responsive health care system, to use institutional and other community-based platforms to spread awareness, mobilize community and facilitate $100 \%$ reporting and review of maternal deaths, to develop and establish a system of continuous client feedback and redressal of grievances, provision of an interdepartmental platform for convergent action plans, effective governance including formulating strategies, supervision and review of the program for corrective actions. All pregnant and lactating mothers till 6 months after delivery with all sick infants are covered under SUMAN.

The key feature of SUMAN is service guarantee to the beneficiaries who are entitled to receive at-least 4 Ante Natal Care (ANC) checkups, one Pradhan Mantri Surakshit Matriva Abhiyan (PMSMA) checkup and six Home Based Newborn Care (HBNC) visits, free transport facility from home to health facility, assured referral services with scope of reaching health facility within 1 hour of any critical case emergency, safe delivery 
by trained health provider, screening to eliminate Mother to Child Transmission of HIV, Hepatitis B and Syphilis with a choice for delayed cord clamping up to delivery of placenta in uncomplicated deliveries, post partum family planning counseling and Information, Education and Communication (IEC) for safe motherhood and Respectful Care with privacy and dignity. Likewise for infants, there is entitlement of early initiation and support for breastfeeding, management of sick neonates, getting zero dose vaccination, birth registration, conditional cash transfers/ direct benefit transfer under various schemes, drop back from institution to home after discharge (minimum $48 \mathrm{hrs}$ ), free and zero expense delivery and Caesarean-section facility in case of complications at public health facilities and time bound redressal of grievances including through a responsive call center/ helpline.

For ensuring the service guarantee, initiatives such as Janani Shishu Suraksha Karyakaram (JSSK), Janani Suraksha Yojana (JSY), Pradhan Mantri Surakshit Matritva Abhiyan (PMSMA), LaQshya, Mothers' Absolute Affection (MAA), care for sick and small babies, home based care for mothers and newborn have been merged under SUMAN. Furthermore, health system strengthening by upgrading infrastructure by establishing Labour and Delivery Room (LDR), Operation Theatres, Obstetric High Dependency Units (HDU)/Intensive Care Units (ICU), Newborn Care Corners, Newborn Stabilization Unit (NBSU), Sick Newborn Care Unit (SNCU), adequate human resource, drugs and diagnostics with creation of centres of excellence for research and quality health services will be ensured.

For implementation of SUMAN, facilities will be identified and notified by the states and district administration will ensure that facilities provide SUMAN Service packages. Afterwards, SUMAN identified facilities are linked through online and IT enabled system and monitored and reviewed every six months for their performance. For reporting of maternal deaths, a centralized toll free number has been created so that every maternal death is reported and investigated.

Earlier, Ministry of Health and Family Welfare launched LaQshya initiative in the year 2017. LaQshya aims at reducing maternal and infant mortality by improving intra partum and immediate post-partum care in the labour rooms and maternity operation theaters, which is where maximum maternal deaths and still births occur. This initiative has been implemented in medical colleges, district hospitals, sub divisional hospitals and high delivery load $\mathrm{CHCs}$ and FRUs.

With such political will and newer quality improvement interventions taken up by Government of India, it can be expected that India will achieve the targets of reduction in maternal and infant mortality in coming years. 